\title{
Three Dimensional Flow Analyses in a Diagonal Type MHD Generator
}

\author{
${ }^{1}$ Triwahju Hardianto, ${ }^{2}$ Nobuomi Sakamoto and ${ }^{3}$ Nob. Harada \\ ${ }^{1,2,3}$ Department of Electrical Engineering, Nagaoka University of Technology \\ 1603-1 Kamitomioka, Nagaoka 940-2188, Japan
}

\begin{abstract}
This paper studied development of the three dimensional flow fields in a diagonal channel MHD generator. A diagonal insulating sidewalls type channel is proposed in this work. The partial-differential equations for the three momentum, the enthalpy and the electrical fields are solved by a finite-difference calculation procedure. The turbulence phenomenon is represented by two-equation turbulence model. Calculations have been made for typical MHD Diagonal channel conditions. The development of the three dimensional flow, current density and power generated is presented. At the end part we conduct a modification by diagonal angle of the channel. It is found that the power generated increase according to increment of diagonal angle.
\end{abstract}

Key words: MHD generator, three dimensional analyses, diagonal channel type

\section{INTRODUCTION}

The performance of the three dimensional flow in an MHD channels is studied. The partial-differential equations for the three momentum, the enthalpy and the electrical fields are solved by a finite difference calculation procedure. Model incorporates the interaction between the flow and electrical fields in MHD channels. It consists of Navier Stokes Equations in parabolic form, Maxwell and Ohm's law equations as they apply to the MHD channel and a two-equation turbulence model ${ }^{[1-3]}$. The model is then applied to high-interaction supersonic flow in a channel. The performance of the three dimensional flow, temperature and electrical fields is presented.

In the present study, the configuration effects of insulating sidewall on the performance characteristics of diagonal-type generators are studied analytically. Three dimensional effects are considered by seeking a solution to the governing partial differential equations. The three dimensional Navier Stokes equations governing the transport of a turbulent compressible fluid are solved coupled with the Maxwell equations for the current distributions ${ }^{[4]}$. The turbulence phenomena are represented by a two-equation turbulence model ${ }^{[5]}$ in which two additional equations are solved, one for the transport of turbulence kinetic energy and the other for its dissipation rate. The diagonal type generators generally have two kinds of sidewalls, i.e., conducting sidewalls and insulating sidewalls. The current and potential distributions in both channels are investigated by three dimensional analysis and detailed comparisons are made ${ }^{[6]}$. In this paper, next section describes the governing equations and solution method. Thereafter describes MHD generator channel, the working gas and the results of an illustrative calculation respectively. Last section is the conclusions of this paper.

\section{MATHEMATICAL MODEL}

The governing three dimensional equations are simplified by making the assumptions given below.

Fluid model: The steady, turbulent, incompressible Navier-Stokes equations for fully developed flow in the cross plane take the following form:

Mass continuity:

$$
\frac{\partial}{\partial x}(\rho u)+\frac{\partial}{\partial y}(\rho v)+\frac{\partial}{\partial z}(\rho w)=0
$$

$\mathrm{x}$ momentum:

$$
\frac{\partial}{\partial x}(\rho u u)+\frac{\partial}{\partial y}(\rho v u)+\frac{\partial}{\partial z}(\rho w u)=-\frac{\partial \tilde{p}}{\partial x}+\frac{\partial \tau_{x x}}{\partial x}+\frac{\partial \tau_{y x}}{\partial y}
$$

y momentum:

$$
\begin{aligned}
\frac{\partial}{\partial x}(\rho u v)+\frac{\partial}{\partial y}(\rho v v)+\frac{\partial}{\partial z}(\rho w v)=-\frac{\partial \tilde{p}}{\partial x} & +\frac{\partial \tau_{x y}}{\partial x} \\
& +\frac{\partial \tau_{y y}}{\partial y}-J_{z} B
\end{aligned}
$$

z momentum:

$$
\begin{aligned}
\frac{\partial}{\partial x}(\rho u w)+\frac{\partial}{\partial y}(\rho v w)+\frac{\partial}{\partial z}(\rho w w)=-\frac{\partial \bar{p}}{\partial z} & +\frac{\partial \tau_{x z}}{\partial x} \\
& +\frac{\partial \tau_{y z}}{\partial y}+J_{y} B
\end{aligned}
$$

where $\rho$ is the fluid density, $u, v, w$ is the velocities in the $x, y$ and $z$ directions, respectively. $\tau_{i j}$ is shear stress in the $i$ direction, on the plane perpendicular to $j$

Corresponding Author: Triwahju Hardianto, MHD and Plasmadynamics Lab., Deprtment.of Electrical Engineering, Nagaoka University of Technology, 1603-1 Kamitomioka, Nagaoka 940-2188, Japan, Tel: 810258466000 Ext. 5339, Fax: 81258479500 
direction. $J$ is the current vector with components $J_{x}, J_{y}$ and $J_{z}$, respectively. $p$ is the pressure field and $B$ is the magnetic field.

Enthalpy:

$$
\begin{aligned}
\frac{\partial}{\partial x}(\rho u h)+\frac{\partial}{\partial y}(\rho v h)+\frac{\partial}{\partial z}(\rho w h) & =-\frac{\partial q_{y}}{\partial y}-\frac{\partial q_{x}}{\partial x}+u \frac{\partial \tilde{p}}{\partial x} \\
& +v \frac{\partial \tilde{p}}{\partial y}+w \frac{\partial \bar{p}}{\partial z}+\frac{J^{2}}{\sigma}+D
\end{aligned}
$$

where

$$
D=\left(\mu_{l}+\mu_{t}\right)\left\{\left[\left(\frac{\partial u}{\partial x}\right)^{2}+\left(\frac{\partial v}{\partial y}\right)^{2}\right]+\left(\frac{\partial u}{\partial y}+\frac{\partial v}{\partial x}\right)^{2}+\left(\frac{\partial v}{\partial x}\right)^{2}+\left(\frac{\partial u}{\partial y}\right)^{2}\right\}
$$

where $h$ is the enthalpy, $q$ is the heat fluxes in the $y$ and $z$ directions, $\sigma$ is the electrical conductivity, $\mu_{l}$ is the laminar viscosity and $\mu_{t}$ is the turbulent viscosity.

Turbulence model: In the present study, we represent the turbulent fluxes as the product of a turbulent viscosity and the gradients of the flow variable. The turbulent viscosity is calculated from the local values of the turbulent kinetic energy, $k$ and its dissipation rate, $\varepsilon$, from the formula

$\mu_{t}=C \mu \frac{\rho k^{2}}{\varepsilon}$

The turbulent fluxes are calculated by the following formulas:

$$
\tau_{i j}=\left(u_{t}+\mu_{l}\right)\left(\frac{\partial u_{i}}{\partial x_{j}}+\frac{\partial u_{j}}{\partial x_{i}}\right)
$$

and

$$
q_{i}=-\left(\frac{\mu_{t}}{\sigma_{h}}+\frac{\mu_{l}}{\sigma_{l}}\right)\left(\frac{\partial h}{\partial x_{i}}\right)
$$

The values of $k$ and $\varepsilon$ are obtained from the solution of the following transport equations:

Kinetic energy of turbulence,

$$
\begin{gathered}
\frac{\partial}{\partial x}(\rho u k)+\frac{\partial}{\partial y}(\rho v k)+\frac{\partial}{\partial z}(\rho w k)=\frac{\partial}{\partial x}\left[\left(\frac{\mu_{t}}{\sigma_{k}}+\mu_{l}\right) \frac{\partial k}{\partial x}\right] \\
+\frac{\partial}{\partial y}\left[\left(\frac{\mu_{t}}{\sigma_{k}}+\mu_{l}\right) \frac{\partial k}{\partial y}\right]+G-\rho \varepsilon
\end{gathered}
$$

dissipation rate

$$
\begin{gathered}
\frac{\partial}{\partial x}(\rho u \varepsilon)+\frac{\partial}{\partial y}(\rho v \mathcal{E})+\frac{\partial}{\partial z}(\rho w \mathcal{E})=\frac{\partial}{\partial x}\left[\left(\frac{\mu_{t}}{\sigma_{\varepsilon}}+\mu_{l}\right) \frac{\partial \varepsilon}{\partial x}\right] \\
+\frac{\partial}{\partial y}\left[\left(\frac{\mu_{t}}{\sigma_{\varepsilon}}+\mu_{l}\right) \frac{\partial \mathcal{\varepsilon}}{\partial y}\right]+\frac{C_{1} G \varepsilon}{k}-C_{2} \rho \frac{\varepsilon^{2}}{k}
\end{gathered}
$$

where $G$ represent the production of the kinetic energy of turbulence due to the interaction of the shear stresses with the velocity gradients. More details of the turbulence model including the treatment of near-wall regions may be found in ${ }^{[4]}$.

Electrical model: Maxwell equations together with Ohm's law are used to solve for the electric field and current density. The electric field, $\boldsymbol{E}$ and the current density $\boldsymbol{J}$ and the cross sectionally averaged generated power $\boldsymbol{P}_{\boldsymbol{0}}$ are obtained by solving the following equations:

$\nabla \times E=0$

$\nabla \bullet J=0$

$J=\sigma(E+u \times B)-\frac{\beta}{B} J \times B$

$P_{o}=\langle J . E\rangle$

From Ohm's law, the current density components, $J_{x}, J_{y}$ and $J_{z}$, can expressed as:

$$
\begin{aligned}
& J_{x}=-E_{z} \sigma \frac{\partial \phi_{1}}{\partial x}-\sigma \frac{\partial \phi_{2}}{\partial x} \\
& J_{y}=E_{z} \sigma_{n}\left(\beta-\cot \gamma-\frac{\partial \phi_{1}}{\partial y}\right)-\sigma_{n}\left[(w-\beta v) B+\frac{\partial \phi_{2}}{\partial y}\right] \\
& J_{z}=E_{z} \sigma_{n}\left(1+\beta \cot \gamma+\beta \frac{\partial \phi_{1}}{\partial y}\right)+\sigma_{n}\left[(\beta w+v) B+\beta \frac{\partial \phi_{2}}{\partial y}\right]
\end{aligned}
$$

where $\phi$ is the electric potential, $\beta$ is the Hall parameter and $\gamma$ is the diagonal angle.

\section{Method}

The solution of the partial differential equation is obtained by MGMHD (multigrid magnetohydrodynamic) method $^{[2]}$. First, the cross-stream plane continuity and momentum equations are solved simultaneously by the full approximation storage block implicit multigrid method. Second, the cross-stream potential equations are solved using a full approximation storage multigrid method.

\section{MHD channel type}

Schematic representation of generator configuration in cartesian coordinate with diagonal insulating sidewalls type is shown in fig. 1 . Where $\mathrm{c}$ is the electrode conductor width and $\mathrm{P}$ is the pitch length. The generator dimensions and operating conditions are presented in table 1 . The channel is $2 \mathrm{~m}$ long and has a square cross section that increases linearly from the channel inlet to the channel exit. The channel inlet and exit are $0.35 \mathrm{~m} \mathrm{x}$ $0.35 \mathrm{~m}$ and $0.7 \mathrm{~m} \times 0.7 \mathrm{~m}$ respectively. The channel has a diagonalization angle of $45^{\circ}$ and insulated sidewalls. The magnetic field is $1.5 \mathrm{~T}$ throughout the channel. The plasma enters the channel uniformly at supersonic velocity and at an inlet pressure $1.58 \times 10^{5} \mathrm{~Pa}$. 
Table 1: $\quad$ Operating conditions

\begin{tabular}{ll}
\hline Mass flow rate $(\mathrm{kg} / \mathrm{s})$ & 35.5 \\
Channel geometry $(\mathrm{m}),($ rectangular cross section) & \\
Length $(\mathrm{m})$ & 2 \\
Inlet height and width $(\mathrm{m})$ & 0.35 and 0.35 \\
Exit height and width $(\mathrm{m})$ & 0.7 and 0.7 \\
Magnetic field strength $(\mathrm{T})$ & 1.5 \\
Inlet flow velocity $(\mathrm{m} / \mathrm{s})$ & 1491 \\
Inlet static Temperature $(\mathrm{K})$ & 3500 \\
Inlet static pressure $(\mathrm{Pa})$ & $1.58 \times 10^{5}$ \\
Wall temperature $(\mathrm{K})$ & 1500 \\
Working fluid & Heavy oil fuel \\
Load current $(\mathrm{A})$ & 3500 \\
Diagonalization angle $(\mathrm{g})$ & $45^{\circ}$ \\
Much Number & 1.6 \\
Wall sand-grain roughness $(\mathrm{mm})$ & 5 \\
\hline
\end{tabular}

Working gas: The working fluid used in the calculation is a combustion product of heavy-oil fuel and oxygen of a stoichiometric factor $1: 1$, where $\mathrm{KOH}$ and $\mathrm{K}_{2} \mathrm{SO}_{4}$ are seeded so that the potassium atom is contained in a ratio of $1 \mathrm{wt} \%$ to the combustion gas. The dependences of the electrical conductivity $\sigma$ and the Hall parameter $\beta$ on the gas temperature $T$, the static pressure $p$ and the applied magnetic field $\mathrm{B}$ were determined by the following equations:

$$
\begin{aligned}
\sigma & =89.9 p^{-0.51} T^{1.055} \exp \left(-2.52 \times 10^{4} / T\right) \mathrm{mho} / \mathrm{m} \\
\beta & =4.43 \times 10^{-4} p^{-0.99} T^{0.97} B
\end{aligned}
$$

\section{SIMULATION RESULTS}

This section describes a performance of diagonal MHD generator with electrical power output and the evolution of the predicted secondary flows and flow asymmetries. The plasma enters the channel uniformly at supersonic velocity and at an inlet pressure of $1.58 \times 10^{5} \mathrm{~Pa}$. The inlet temperature is $3500 \mathrm{~K}$ and the wall temperature is maintained at $1500 \mathrm{~K}$. The magnetic is $1.5 \mathrm{~T}$. The generator is electrically loaded at $3500 \mathrm{~A}$, which gives a global electrical power output of 7.79 MW. Figure 2 shows the predicted development of the transverse cross-sectional plane velocities at four axial locations along the generator channel. The velocity flow performance diverges outwardly near the core region to the walls and to the electrodes. The divergence is not at the center of the channel cross-section, but at the close of center (Fig. 2) at $\mathrm{z}=0.5 \mathrm{~m}$. As the flow progress downstream, the center of divergence moves toward center of the channel at $\mathrm{z}=1.0 \mathrm{~m}$ and two vortex pattern in the left and right sides of the channel are created since symmetry is assumed.

Further down the channel where magnetohydrodynamic interactions became weaker at exit channel, the flow continues to diverge towards the duct walls to keep strong secondary velocities in the side wall regions at $\mathrm{z}=1.5 \mathrm{~m}$ and $2 \mathrm{~m}$.

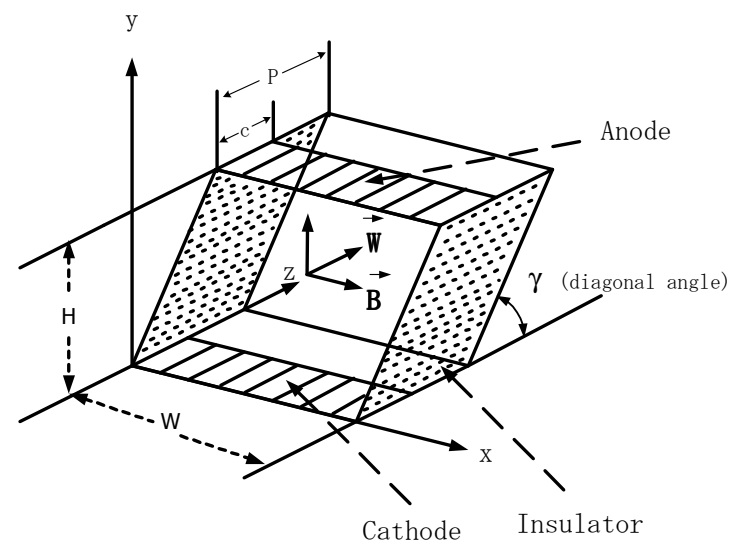

Fig. 1: Schematic of diagonal insulating sidewalls
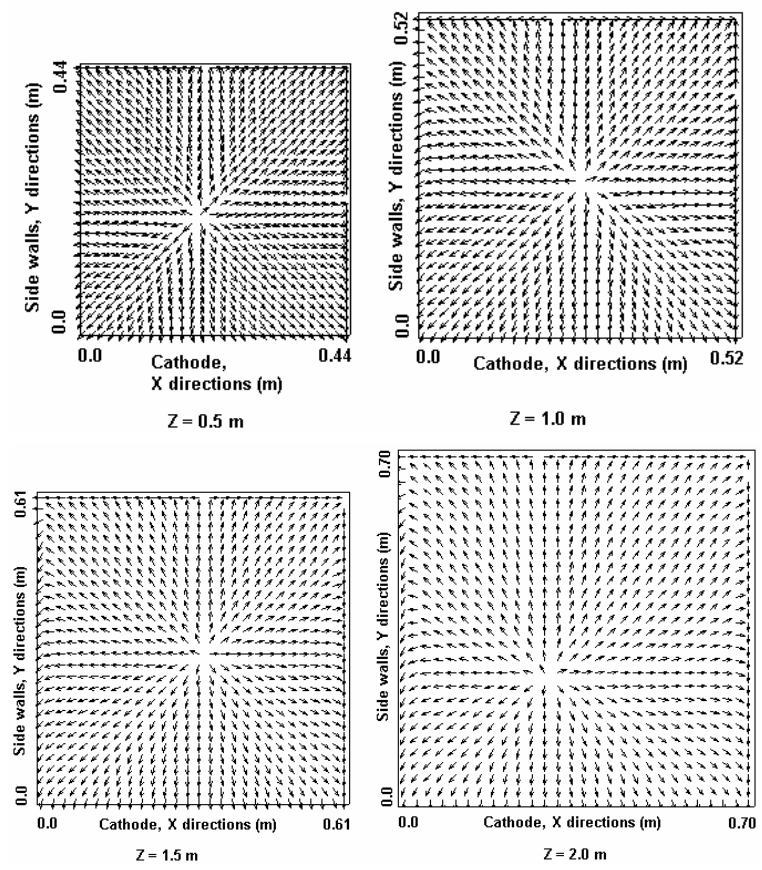

Fig. 2: Development of secondary flow vortices

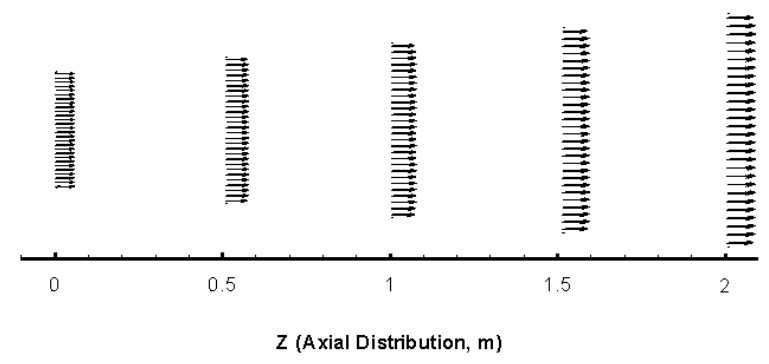

Fig. 3: Development of fluid flow in the central axial cross section

An axial cross section of the flow development in the plane of symmetry is shown in Fig. 3. The flow does not separate and is supersonic throughout the channel.

A illustrative surface of the axial velocity distribution is shown in Fig. 4, by means of velocity 

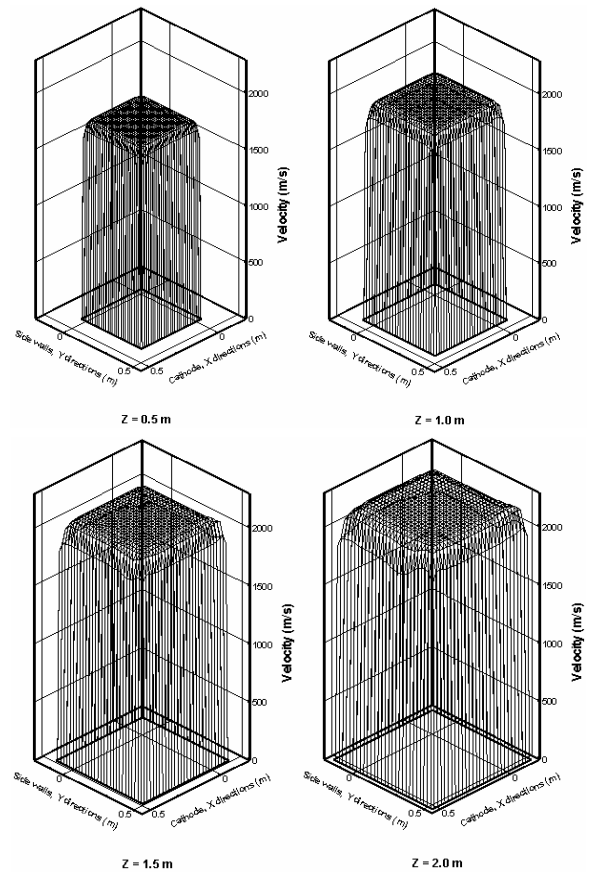

Fig. 4: Axial Flow Development in a diagonal generator
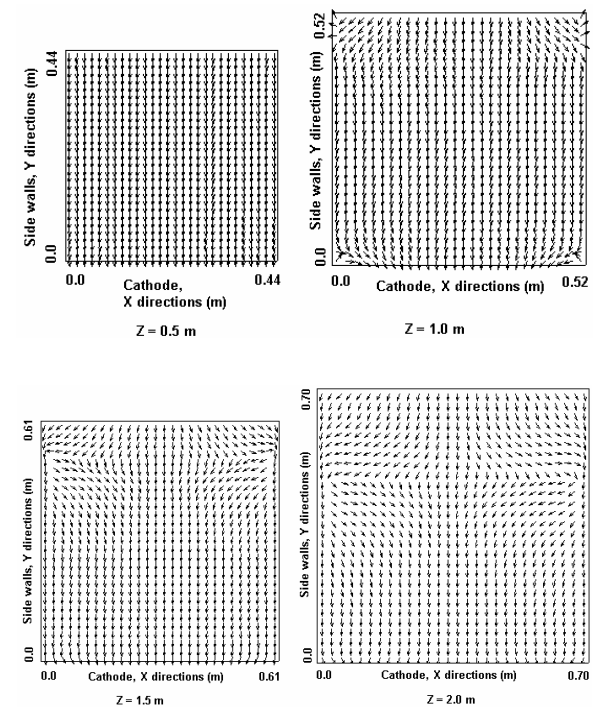

Fig. 5: Development of the transverse cross-sectional current density in a diagonal generator

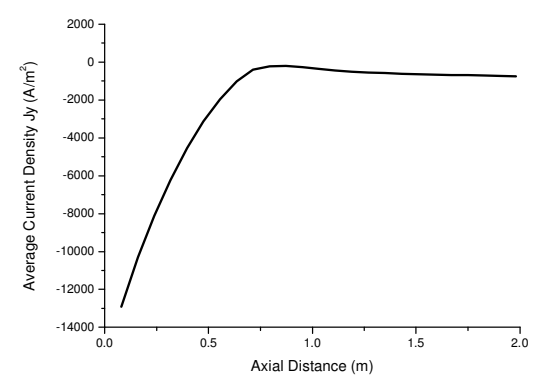

Fig. 6: Distribution of average current density

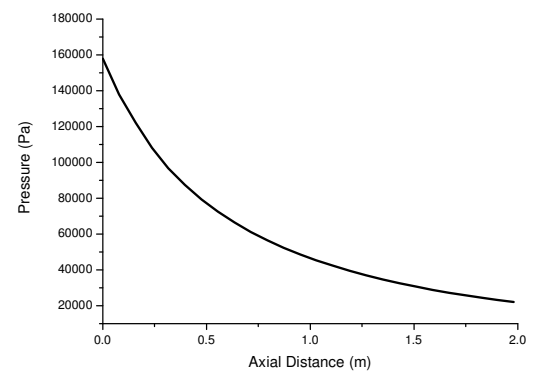

Fig. 7: Distribution of pressure

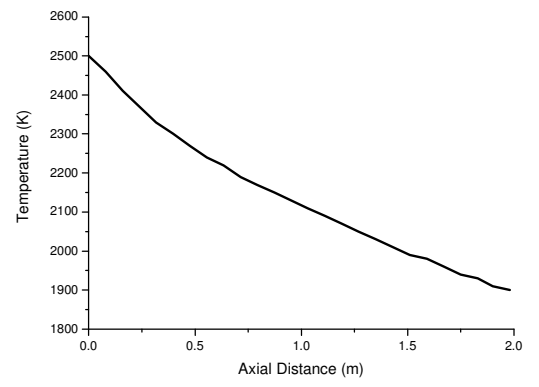

Fig. 8: Distribution of temperature

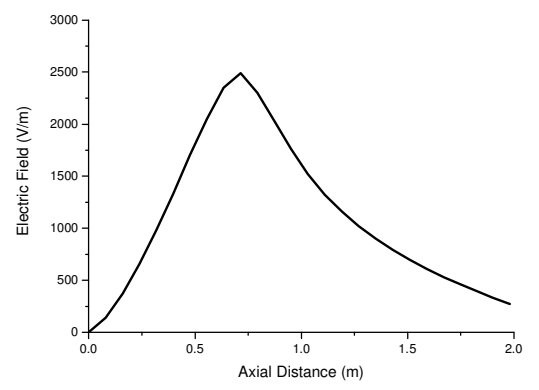

Fig. 9: Distribution of electric field

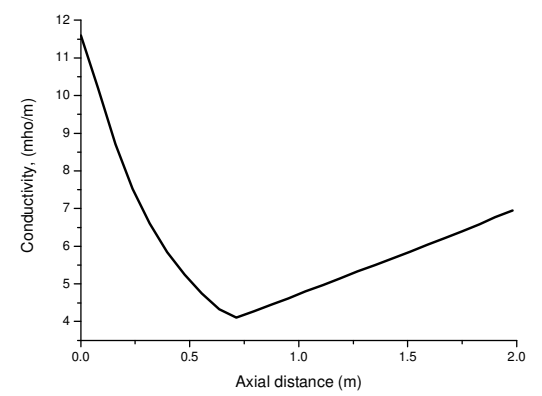

Fig. 10: Distribution of conductivity

surface plots. In the sidewalls boundary layers, small velocity overshoots are observed indicating that the strong secondary flows suppress any flow field distortions that could develop in the transverse cross section of the channel. Thus, strong secondary flows tend to establish almost uniform cross-sectional 


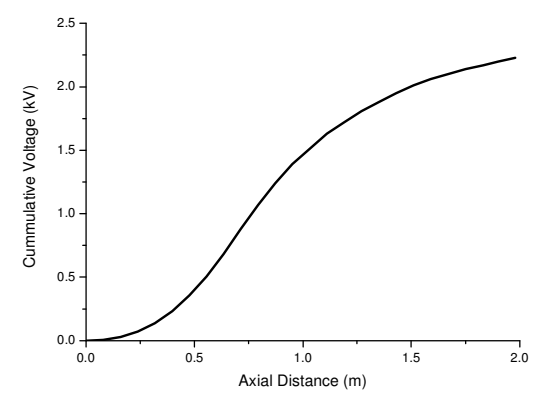

Fig. 11: Distribution of cumulative voltage

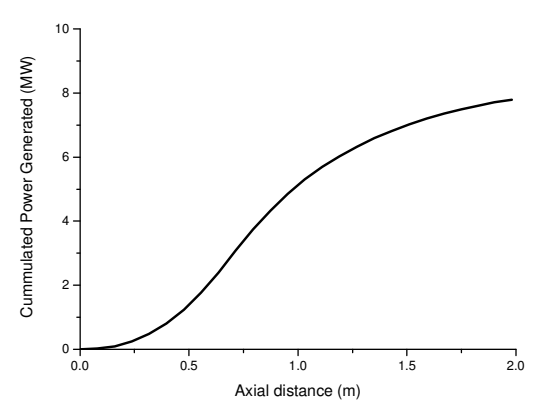

Fig. 12: Distribution of cumulated power generated

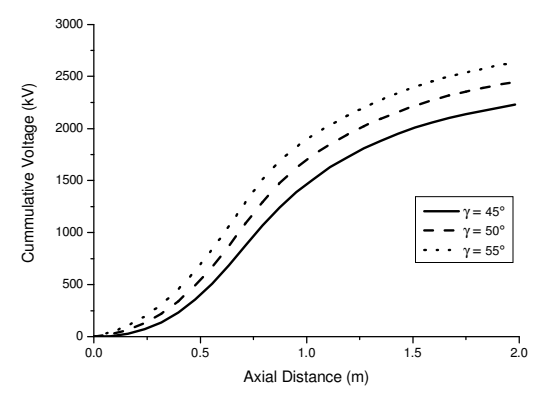

Fig. 13: Distribution of cumulated voltage with diagonal angle modification

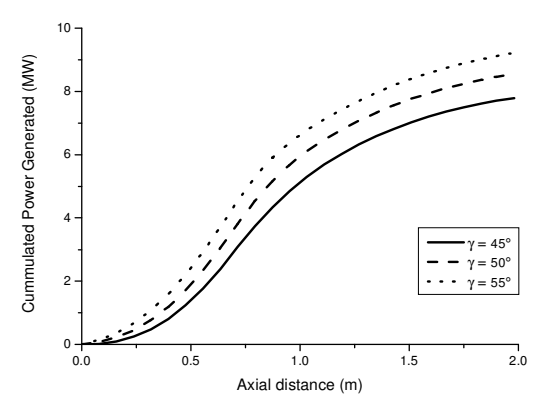

Fig. 14: Distribution of cumulated power generated with diagonal angle modification Diagonal angle modification distributions of axial velocity, which improve the performance of the generator and limit instabilities by current constrictions. The emergence of velocity overshoots results from the imposition of nonuniform magnetic forces $(J y \bullet B)$ on the flow field. The flow enters the channel at supersonic velocity and gradually accelerates during its expansion along the channel. In this generator, the axial flow is more accelerated than in the cathode, anode and sidewalls regions.

Figure 5 shows the development of the cross sectional current density at different axial locations. The faraday current density Jy develops uniformly across the transverse section of the channel. In Fig. 6, the current density Jy is negative throughout the channel cross section. The current density attains a peak $12900 \mathrm{~A} / \mathrm{m}^{2}$ that correspond to conductivity and the magnetic field strength.

Figure 7 shows the axial pressure along the channel. In the case, the static pressure near the channel inlet is $1.58 \times 10^{5}$ pa and about $0.22 \times 10^{5}$ pa at the channel exit. The performance indicates that is not strong enough to influence the gas dynamic characteristics of the channel. The corresponding axial centerline static temperature along the channel is shown in Fig. 8. The inlet channel core static temperature is $2500 \mathrm{~K}$, which drops rapidly to $1900 \mathrm{~K}$ at the channel exit because of the supersonic flow expansion in the channel.

The corresponding Hall electric fields and conductivity variations along the channel are plotted in Fig. 9 and 10. The electric field reaches a maximum value of $2490 \mathrm{~V} / \mathrm{m}$ at $\mathrm{z}=0.714 \mathrm{~m}$ and then decreases along the channel, as the plasma conductivity drops because of the expanding supersonic flow along the channel.

The electric voltage and power generated shown in Fig. 11 and 12 rise uniformly along the channel. The voltage increases at the channel exit of $2.23 \mathrm{kV}$, which results in an electric power output of 7.79 MW with using load current $3500 \mathrm{~A}$.

In this section, diagonal angle modification of MHD generator is presented. Modification is made with changing diagonal angle of $45^{\circ}, 50^{\circ}$ and $55^{\circ}$ at the MHD generator channel. On these results, we obtain that the electric voltage and power generated are increase according to increment of diagonal angle as shown in Fig. 13 and 14.

\section{CONCLUSION}

In the present paper, the three dimensional flow in a diagonal insulating sidewalls type of MHD generator channel has been studied by solving the governing partial-differential equations for the coupled electrical and flow fields. Performance of velocity, current density, pressure, temperature, voltage and power 
generated are found. And the power generated is increase according to increment of diagonal angle.

\section{REFERENCES}

1. Hardianto, T., N. Sakamoto and N. Harada, 2006. Three dimensional flow analysis in an MHD generator. New Energy Technology Symposium, Tsukuba, Japan.

2. Bouillard, J.X., J.L. Krazinski, S.P. Vanka and G.F. Berry, 1989. User's Manual For MGMHD: A Multigrid Three-Dimensional Computer Code for The Analysis of Magnetohydrodynamic Generators and Diffusers. Argonne National Laboratory
3. Vanka, S.P. and R.K. Ahluwalia, 1982. Three dimensional flow and thermal development in magnetohydrodynamic channels. J. Energy, 6: 218-221.

4. Vanka, S.P. and R.K. Ahluwalia, 1983. Coupled three dimensional flow and electrical calculations for faraday MHD generators. J. Energy, 7: 65-72.

5. Launder, B.E. and D.B. Spalding, 1974. The numerical computation of turbulent flows. Computer Methods in Applied Mechanics and Engineering, 3: 269-289.

6. Hara, T. and J. Umoto, 1979. Three-dimensional effects of electrode configuration on diagonal MHD generator performance. J. Energy, 3: 16-22. 